\title{
Transients in oxygen outflow above the polar cap as observed by the Cluster spacecraft
}

\author{
H. Nilsson ${ }^{1}$, M. Waara ${ }^{1}$, O. Marghitu ${ }^{2,3}$, M. Yamauchi ${ }^{1}$, R. Lundin ${ }^{1}$, H. Rème ${ }^{4}$, J.-A. Sauvaud ${ }^{4}$, I. Dandouras ${ }^{4}$,

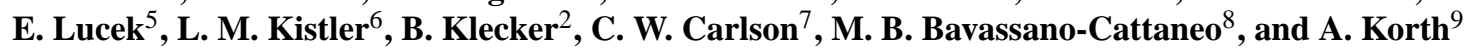 \\ ${ }^{1}$ Swedish Institute of Space Physics, Kiruna, Sweden \\ ${ }^{2}$ Max-Planck-Institut für Extraterrestriche Physik, Garching, Germany \\ ${ }^{3}$ Institute for Space Sciences, Bucharest, Romania \\ ${ }^{4}$ Centre d'Etude Spatiale des Rayonnements, Toulouse, France \\ ${ }^{5}$ Imperial College of Science, Technology and Medicine, London, UK \\ ${ }^{6}$ University of New Hampshire, Durham, USA \\ ${ }^{7}$ Space Science Laboratory, University of California, Berkeley, USA \\ ${ }^{8}$ Istituto di Fisica dello Spazio Interplanetario, Rome, Italy \\ ${ }^{9}$ Max-Planck-Institut für Sonnensystemforschung, Katlenburg-Lindau, Germany
}

Received: 4 January 2008 - Revised: 17 June 2008 - Accepted: 10 September 2008 - Published: 21 October 2008

\begin{abstract}
Oxygen ion outflow associated with the cusp and cleft give rise to persistent oxygen ion beams which can be observed over the polar cap. For high altitude spacecraft such as Cluster these beams are often observed for several hours on each occasion. This allows for a study of typical temporal structures on the time scale of minutes. We have used 3 years of data from spring, January to May of years 2001 to 2003, for a study of the oxygen number flux variation in the polar cap ion outflow. The source of these oxygen ion beams is the cusp and cleft, and variations in ionospheric upflow on time scales of around $8 \mathrm{~min}$ have been reported from ground based studies using incoherent scatter radar. Such upflows typically do not reach escape velocity, and further energization above the ionosphere is required for outflow to occur. Our study shows that a typical time scale between sudden number flux enhancements observed by Cluster in a geocentric distance range of $5 R_{E}$ to $12 R_{E}$ is 5 to $10 \mathrm{~min}$. A superposed epoch study does not reveal any significant convection velocity or temperature changes around the flux enhancement events. Sudden temperature enhancements occur with a typical time interval of about 4 min, A superposed epoch study does not reveal any number flux enhancements associated with the temperature enhancements. The clear modulation of the high altitude number flux in a manner which resembles the modulation of the ionospheric upflow indicates that this is the main limiting factor determining the total outflow.
\end{abstract}

Correspondence to: H. Nilsson

(hans.nilsson@irf.se)
The process behind transient upflow events in the ionosphere is therefore important for the total ionospheric outflow. Subsequent heating above the ionosphere appears to be common enough in the cusp/cleft region that it does not significantly modulate the oxygen ion number flux.

Keywords. Ionosphere (Ionosphere-magnetosphere interactions) - Magnetospheric physics (Magnetopause, cusp, and boundary layers; Polar cap phenomena)

\section{Introduction}

Heavy ion escape from the Earth's ionosphere appears to take place in a two step process. First an upflow from the ionosphere occurs. The flows are typically of the order of $100 \mathrm{~m} / \mathrm{s}$ to $1000 \mathrm{~m} / \mathrm{s}$, and the ion velocity does not reach escape velocity. In order to escape the ions must also experience further energization at higher altitude. It is not obvious if the ionospheric upflow or the subsequent energization is the most important process in determining the total number flux of outflowing heavy ions. The upflows are often associated with energy input into the ionosphere through enhanced magnetospheric electric fields and particle precipitation. Changing high altitude boundary conditions, i.e. the pressure profile of the plasma above the ionosphere, may also play an important role. Yau and André (1997) provides a recent review of the mechanisms involved in ion outflow.

Published by Copernicus Publications on behalf of the European Geosciences Union. 
One of the most prominent sources of heavy ion outflow is the cusp/cleft and polar cap region. The ionospheric upflow there is strong due to both soft particle precipitation which strongly enhances the F-region electron temperature (Nilsson et al., 1994, 1998; Ogawa et al., 2003) and due to strong magnetospheric electric fields (Schunk et al., 1975). Mid altitude $\left(3-5 R_{E}\right)$ satellites have identified the same region as the source of the cleft ion fountain (Lockwood et al., 1985). Further up along the field line subsequent heating is typically observed, for recent Cluster observations see Bouhram et al. (2004); Nilsson et al. (2006); Arvelius et al. (2005). Already at low altitudes such as at the Freja spacecraft altitude of $1700 \mathrm{~km}$ significant ion heating is observed (Norqvist et al., 1998). Because further wave heating in the cusp/cleft region seems fairly common it is possible that this is not a limiting factor. The number flux of ionospheric origin ions should then mainly depend on the ionospheric upflow.

Ionospheric upflow in the cusp/cleft region has been studied by incoherent scatter radars, both Søndre Strømfjord (e.g Nilsson et al., 1998) and EISCAT Svalbard Radar (e.g. Ogawa et al., 2003). In the latter paper the upflow region observed by the EISCAT Svalbard incoherent scatter radar was connected to a dayside magnetospheric source region identified using DMSP satellite data. They found that the upflow occur both in the cusp (as reported by Nilsson et al., 1998) but also in the mantle region, just poleward of the cusp. Mid altitude satellite measurements have traced the source of mid altitude oxygen ion beams to the cusp region (Øieroset et al., 1999; Bouhram et al., 2004; Dubouloz et al., 1998), and also high altitude polar cap oxygen beams have been traced to the cusp region (Nilsson et al., 2004). Several of the mid altitude studies indicate that it is just a latitudinally narrow region of $1-2^{\circ}$ which contributes strongly to the heating (Knudsen et al., 1994; Dubouloz et al., 1998). This indicates that magnetospheric conditions could influence and modulate the high altitude oxygen ion number flux, as upflow appears to occur in a wider latitudinal region than the strongest subsequent heating.

Satellite investigations of oxygen ion outflow can usually not study flux variations on a few minute time scale. Low and mid altitude spacecraft traverse a region where outflowing ions are seen too rapidly to observe such modulations. Spatial and temporal effects can usually not be separated. In the ionosphere incoherent scatter radars can study outflow variations on time scales of a few minutes. Moen et al. (2004) could show using EISCAT radar and optical data that there was a one to one relationship between poleward moving auroral forms and ionospheric upflow in the cusp region. Poleward moving auroral forms are transient auroral forms in the cusp which recur with a frequency similar to what is expected from flux transfer events (Elphic et al., 1990; Øieroset et al., 1996), i.e. in the 5 to 10 min range.

We have used Cluster data from the high altitude (about 5$12 R_{E}$ geocentric distance) polar cap to study the oxygen ion number flux variation. An algorithm has been used to find sudden enhancements of the oxygen ion number flux. A statistical distribution of the time interval between number flux enhancements is derived from a data set of 3 years of high altitude Cluster polar cap data (obtained in January to May years 2001 to 2003) from one of the Cluster spacecraft. The relation to electric fields and ion temperature enhancements in the time interval around the oxygen flux enhancements is investigated using a superposed epoch method.

\section{Observations}

\subsection{Observational techniques}

We use data from the Cluster Ion Spectrometer (CIS) on board Cluster spacecraft 4 . The CIS instrument is described in detail in Rème et al. (2001). CIS consists of two different ion spectrometers, COmposition and DIstribution Function analyzer (CODIF) which can resolve the major magnetospheric ions and Hot Ion Analyzer (HIA) which has no mass resolution but higher angular and energy resolution. We will only present results from the CODIF instrument.

CODIF can resolve $\mathrm{H}^{+}, \mathrm{He}^{++}, \mathrm{He}^{+}$and $\mathrm{O}^{+}$through a time of flight technique. The detector has a field of view of $360^{\circ}$ orthogonal to the spin plane, divided into 16 sectors of $22.5^{\circ}$ each. The angular resolution is likewise $22.5^{\circ}$ in the spin plane. The energy coverage in the modes of interest to us is from $15 \mathrm{eV}$ per charge up to $38 \mathrm{keV}$ per charge in 16 or 31 logarithmically spaced steps with a $\Delta E / E$ of 0.16 . Due to some instrument problems the practical lower limit is often $40 \mathrm{eV}$. We have therefore only used data above $40 \mathrm{eV}$.

The ion outflow we are discussing in this paper is usually in the form of a cold ion beam, i.e. the bulk velocity is usually higher than thermal velocity (see Fig. 1 for a sample spectrogram, as well as Nilsson et al., 2004, 2006, for details). Typically all observed oxygen ions are flowing out, except for in a few heating cases at the highest altitudes. Therefore the limited resolution of the instrument is not crucial for the determination of the outflow. There may exist some ambiguity between field-aligned and field-perpendicular drift due to limited angular resolution. This will not result in any artificial sudden changes of the estimated field-aligned drift.

The CODIF instrument has two sensitivity ranges, one low sensitivity mode for the solar wind and a high sensitivity mode for the magnetosphere. The calibration of the instrument in principle takes such changes into account, but $\mathrm{O}^{+}$ ions are often brought into the measurable range when the instrument changes to high sensitivity mode, so that a sudden enhancement may erroneously be detected. This will only happen when the spacecraft enters the nominal magnetosphere and therefore only once per orbit. Almost all our data was obtained in the high sensitivity mode.

We also use data from the Cluster fluxgate magnetometers (Balogh et al., 2001). The magnetic field data was used to calculate field aligned and field perpendicular bulk velocities 


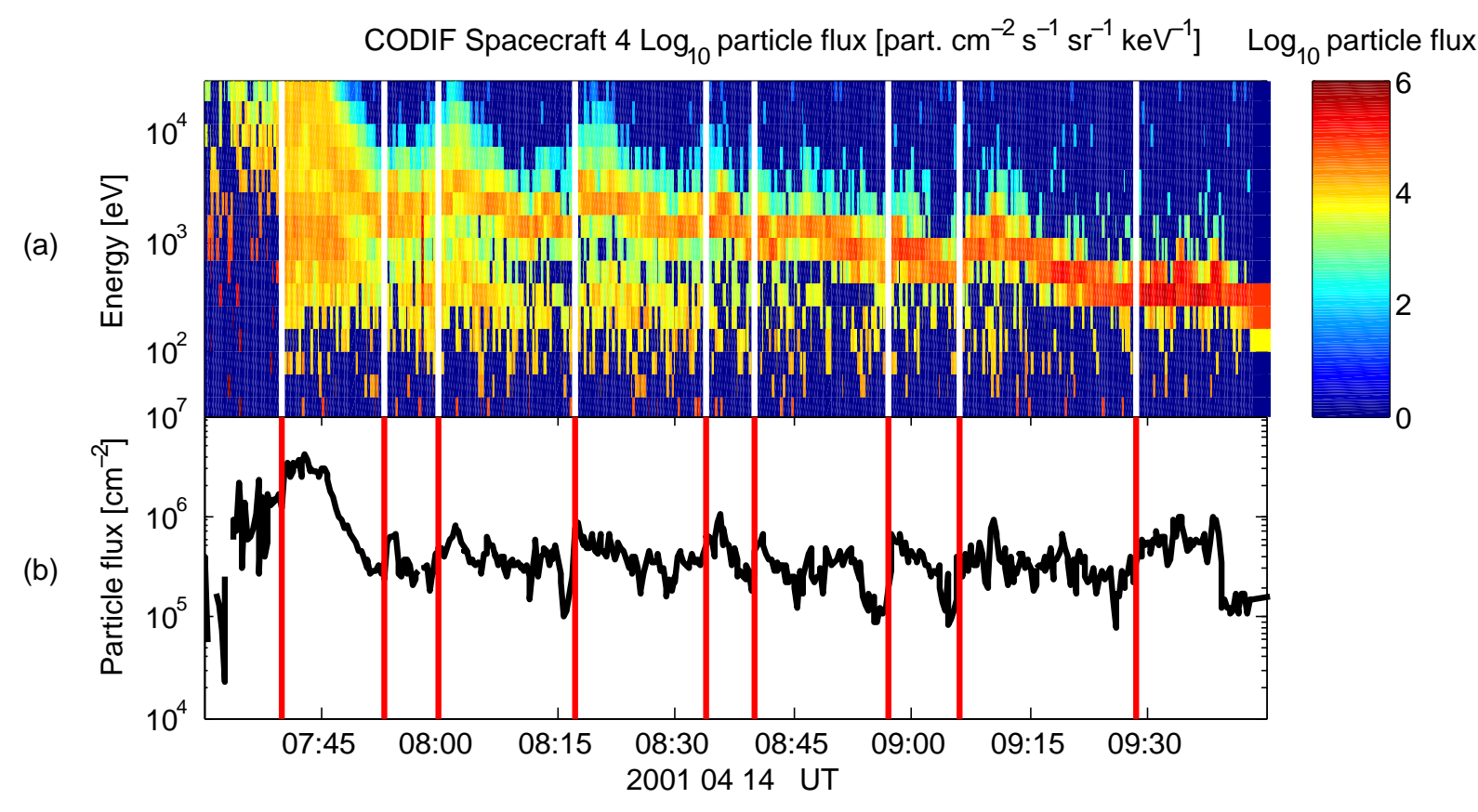

Fig. 1. Oxygen ion data from Cluster spacecraft 4, taken on 14 April 2001. (a) Omnidirectional differential particle flux of $\mathrm{O}^{+}$ $\left[\mathrm{cm}^{-2} \mathrm{~s}^{-1} \mathrm{sr}^{-1} \mathrm{keV}^{-1}\right]$. Automatically detected number flux enhancement events are indicated with vertical white lines. (b) Line plot of the $\mathrm{O}^{+}$field aligned number flux $\left[\mathrm{cm}^{-2}\right]$, calculated as the integrated total flux for particles above $40 \mathrm{eV}$. All flux is outward, i.e. away from Earth.. Automatically detected number flux events are indicated with vertical red lines.

and temperatures. All moments are integrations over the measured distribution function.

\subsection{Data set}

The data set consists of all $\mathrm{O}^{+}$beams clearly seen in the energy spectrograms of the CODIF data of Cluster 4 for spring (January to May) of the years 2001 to 2003. This corresponds to high altitude passes over the polar cap in both hemispheres. Most of the ion beams were observed at geocentric distances between 5 and $12 R_{E}$. The beams were identified through visual inspection of energy spectrograms. A sample spectrogram is shown in Fig. 1, panel (a). Only events lasting more than $1 \mathrm{~h}$ were selected, and such events were seen in about two-thirds of the orbits. The data represents the majority of ion observations in the high altitude polar cap, but there may be additional outflow resulting in more transient oxygen observations at the polar cap altitude.

All spectrograms are rather similar, with a relatively cold population at lower altitude/higher latitude and higher energies and temperatures (wider energy spectrograms) at high altitude/lower latitude. The geometry of the observations is indicated in Fig. 2, reproduced from Nilsson et al. (2006).

From Fig. 2 one can see that magnetic local time is not a very meaningful parameter for this high altitude high latitude data set. The magnetic local time can vary very much along both a convection stream line and along the spacecraft orbit, without this actually indicating anything else than the fact that the observations occur around the pole. Also the invariant latitude is of limited use, it is the distance to the source which is important. Lower altitude spacecraft may pass the source and one may recast the observations into dynamic boundary coordinates, e.g. Andersson et al. (2005), centered on the source region (the cusp). Our observations are mainly from the mantle, just poleward of the cusp. The $\mathrm{H}^{+}$ions observed simultaneously with the $\mathrm{O}^{+}$ions are typically also flowing out. These are mainly magnetosheath origin ions which have mirrored in the Earth's magnetic field. The time resolution of the ion data varies between 4 and $16 \mathrm{~s}$, depending on telemetry available at the time of downlink.

Intense fluxes of $\mathrm{H}^{+}$affects the CODIF instrument in such a way that some of the $\mathrm{H}^{+}$counts are erroneously registered as $\mathrm{O}^{+}$counts. To remove data when contamination from the $\mathrm{H}^{+}$channel significantly affects the $\mathrm{O}^{+}$channel we employ the same method as was used and described in Nilsson et al. (2006). Contamination occur in the same energy channels as the most intense $\mathrm{H}^{+}$fluxes, so the energy distribution of the contamination will follow that of the original $\mathrm{H}^{+}$flux, though at much lower intensity. Velocity related moments interpreting this data as $\mathrm{O}^{+}$will yield bulk velocities one fourth of the original $\mathrm{H}^{+}$bulk velocities due to the mass difference between the two ion species. If all counts in the $\mathrm{O}^{+}$channel are due to contamination from $\mathrm{H}^{+}$the perpendicular bulk velocity of $\mathrm{O}^{+}$will therefore have precisely one fourth the value for $\mathrm{H}^{+}$. The perpendicular bulk drift velocity is dominated by the $\boldsymbol{E} \times \boldsymbol{B}$ drift which is the same for both species, 


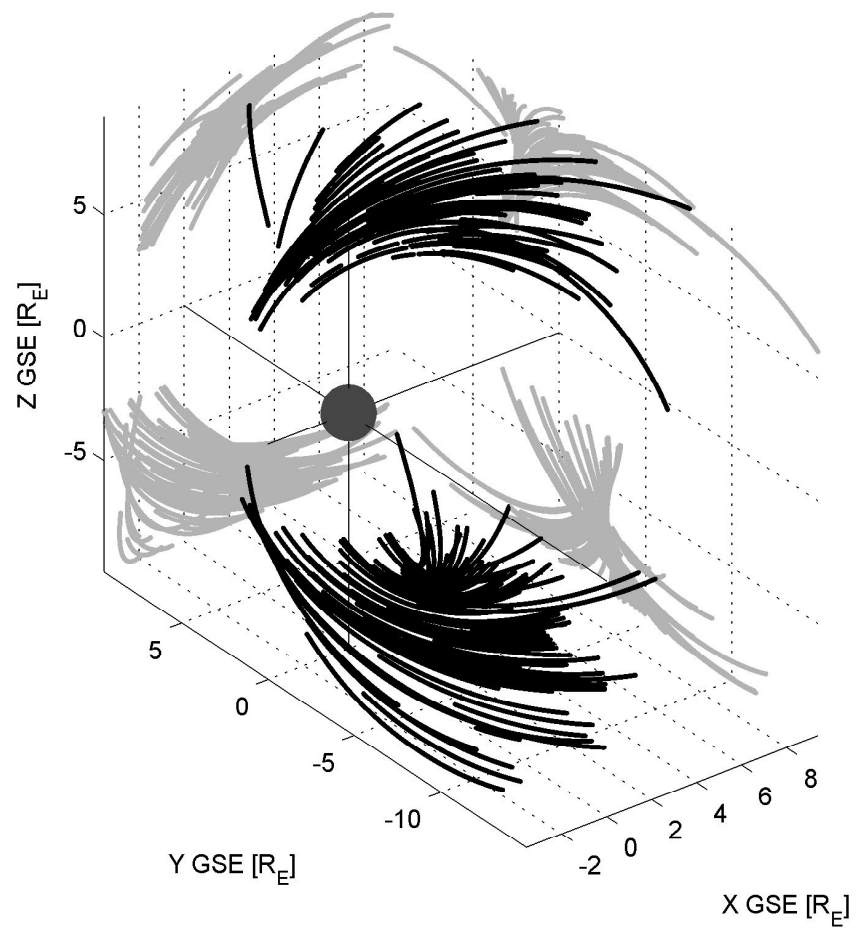

Fig. 2. Distribution of data points used in the study. Orbits are shown in black, projections on the Y-Z and X-Z planes are shown with grey lines. The axes are Geocentric Solar Ecliptic (GSE) coordinates in units of $R_{E}$. The sun is towards positive $\mathrm{x}$.

so the occurrence of the one to four relation in the perpendicular bulk drift is a certain sign of significant contamination. See Nilsson et al. (2006) for more details on the removal of this contamination.

\section{Analysis methods}

\subsection{Detection of sudden enhancements}

In order to detect sudden enhancements in the data parameters an automatic algorithm was developed. An average value has been calculated for a specified time window before and after each measurement point. We have tried several different time windows, from $32 \mathrm{~s}$ to $300 \mathrm{~s}, 120 \mathrm{~s}$ was used in the results presented in this paper. Sudden enhancements have been defined as a local maximum of the ratio of the average flux in the window following each data point to the average flux in the window preceding each data point. This ratio has been low pass filtered to remove noise and only contain major local peaks. A forward and reverse filtering algorithm was used to ensure zero phase shift of the filtered signal. Zero crossings of the difference of the filtered time signal were used to detect local extreme points. The change of sign around the zero crossing determines if it is a local maximum or minimum. Finally it was checked that the local maximum corresponds to a relative increase of the parameter above a threshold. The threshold was varied between $20 \%$ and $200 \%$. It was found that the result did not depend much on the chosen threshold value up to an increase of $100 \%$. Above that the number of detected transients decreased significantly. We have used a threshold of $50 \%$ in the calculations presented in this paper.

The sudden enhancement detection has been performed for density, parallel velocity, parallel and perpendicular temperature and the number flux. The number flux is the net total of the flux. For oxygen this is always outward, essentially all observed oxygen ions are moving outward. We will discuss the sudden enhancements in number flux, as this is what we are primarily interested in. No amount of magnetospheric acceleration can increase the total number flux, so modulations of the number flux corresponds to modulations of the source. We use only data for particles above $40 \mathrm{eV}$, so magnetospheric acceleration and heating may bring more particles into the instruments detectable energy range. Therefore it is particularly important to look also at the perpendicular temperature enhancement, indicating local heating which may bring more particles into the instrument measurement range.

In the algorithm determining the time interval between the identified enhancements we also included a correction for the spacecraft motion. The time it would take the currently observed convection to move between consecutive spacecraft positions was added (subtracted) from the time between each consecutive data point. This did not make a visible difference to the final distribution of the time between events.

An example of an ion beam with marked enhancement events is shown in Fig. 1. The energy of the particles is dominated by the drift energy. The energy spectrogram with a flux peak covering only a few energy channels corresponds to a drifting cold population.

As can be seen in Fig. 1, panels a and b, the automatic detection algorithm is quite efficient. A few peaks which one may have picked out by hand are omitted. Lowering the window size or the threshold value would have made these enhancements to be included. Our results show that for the total statistics the precise value of the window size and the threshold value are not very important. A total of 1631 number flux enhancement events were detected, and 2815 perpendicular temperature enhancements.

\subsection{Superposed epoch analysis}

Using the enhancement events detected by the algorithm above, a superposed epoch analysis has been made. Data in a window plus minus $120 \mathrm{~s}$ around each detected event has been normalized to the range 0 to 1 , and interpolated to the same time steps $(4 \mathrm{~s})$ relative to the event time. Zero of the normalized values corresponds to zero of the original value, not to the minimum value within the window (which is an alternative normalization which emphasizes the shape 
Time between number flux enhancement events

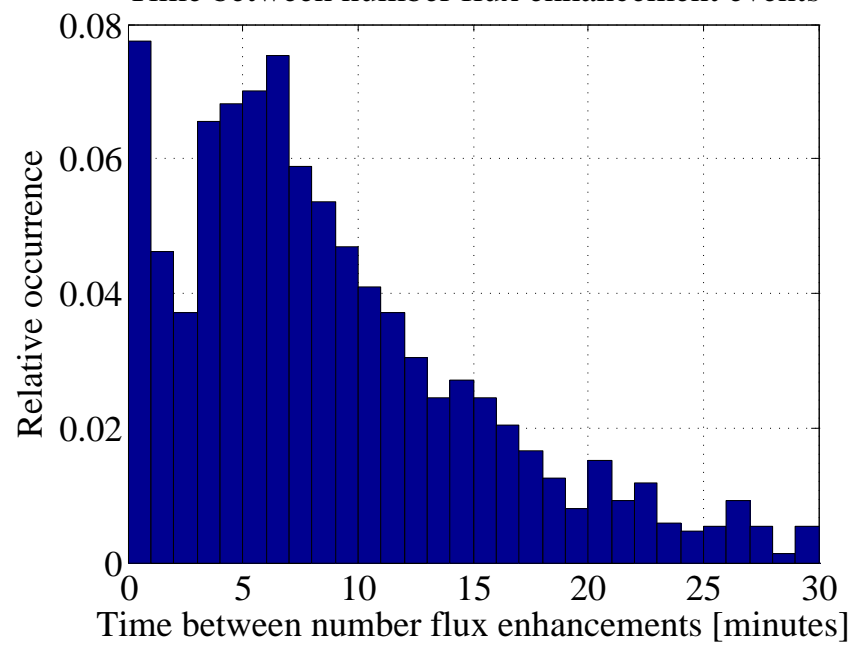

Fig. 3. Distribution of the time interval between number flux enhancement events. The $\mathrm{x}$-axis shows the time between number flux enhancement events [minutes]. The y-axis shows the occurrence frequency.

of the profile but does not allow for a comparison of the relative increase of the parameter values). All normalized data points have then been averaged for each time step relative to the event. This has been made for number flux enhancement events, perpendicular temperature enhancement events and parallel velocity enhancement events. The latter will not be shown in the paper. The $95 \%$ confidence interval of each point of the superposed epoch has been calculated assuming a normal distribution of the data and is indicated with error bars in the plots shown in Sect. 4. A superposed epoch analysis of the proton number flux around the oxygen number flux events was also made (not shown). This showed that the proton flux did not change around the oxygen number flux enhancement. Therefore there is no reason to believe that contamination from protons may in any way affect the transient analysis of the oxygen flux. For the perpendicular temperature the normalized average value turned out to be quite low, without the normalized standard deviation being larger than for the other parameters. This indicates that a few high values, real or spurious, affects the normalization. We therefore performed a noise removal on the perpendicular temperature. Values more than two standard deviations from the mean in the superposed epoch window were discarded. This did not change the qualitative result of the superposed epoch analysis, but increased the average value and made the change of the perpendicular temperature easier to discern.

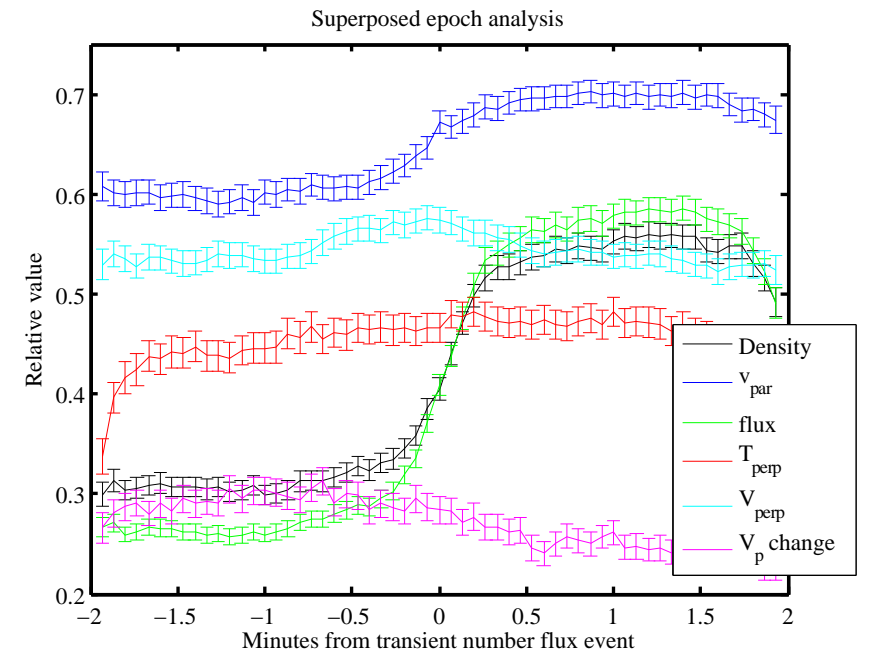

Fig. 4. Superposed epoch analysis of enhanced number flux events. For each event the values have been normalized to the range 0 to 1 by dividing with the maximum value in the superposed epoch window of $\pm 2 \mathrm{~min}$. The $\mathrm{x}$-axis shows the time relative to the enhanced number flux events [minutes]. The y-axis shows the normalized value of the following parameters: Black: Density, Blue: parallel velocity, Green: number flux, Red: perpendicular temperature, Cyan: magnitude of perpendicular velocity and Magenta: magnitude of vector change of perpendicular velocity.

\section{Results}

\subsection{Number flux enhancements}

A histogram of the time between number flux enhancement events is shown in Fig. 3. As can be seen, there are two clear and distinct peaks. The first is in the lowest bin and is the noise peak. More low pass filtering and higher thresholds (we used a $50 \%$ increase threshold) will reduce this peak. The noise peak does not dominate and the noise does not significantly affect the results. The second peak is at $7 \mathrm{~min}$. The interval between 5 and 10 min contains $40 \%$ of the time intervals between events. Increasing the interval to 4 to $13 \mathrm{~min}$ yields an interval which contains half of all the time intervals between events. The resulting histogram does not depend strongly on the length of the window used (we tried from $32 \mathrm{~s}$ to $320 \mathrm{~s}$ and used $120 \mathrm{~s}$ in the final plot). Neither did the result strongly depend on the threshold value discussed in Sect. 3.1, as long as it was kept below $100 \%$ increase. The result of the superposed epoch analysis of the enhanced number flux events is shown in Fig. 4. That the number flux (green line) shows a clear increase around the transient event time shows that the detection algorithm works. The density (black line) is the parameter showing the second largest response. The parallel velocity (blue line) also increases on average, but not as much as density. The magnitude of the perpendicular velocity (cyan) is weakly enhanced at the event but barely above the $95 \%$ confidence limit indicated by the 
Time between perpendicular temperature enhancement events

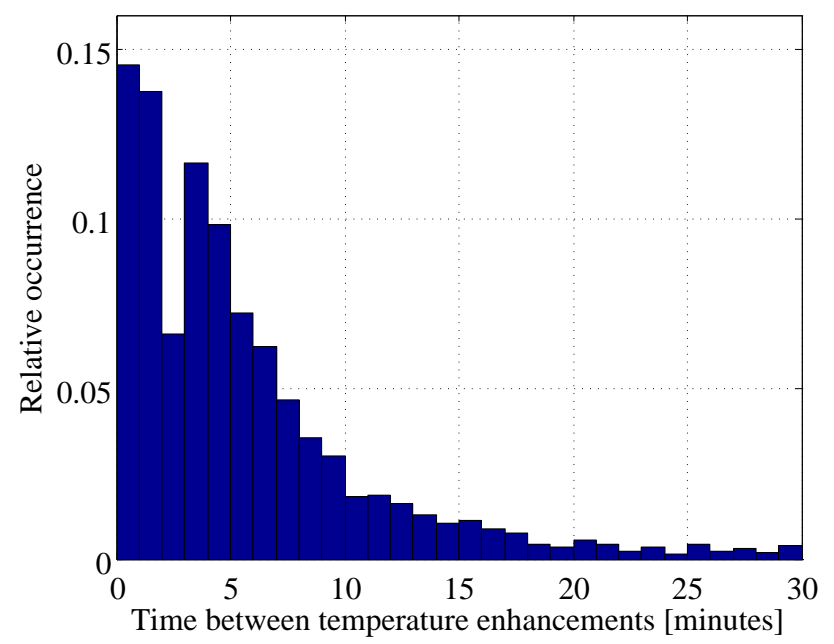

Fig. 5. Distribution of the time interval between perpendicular temperature enhancement events. The $\mathrm{x}$-axis shows the time between temperature enhancement events [minutes]. The y-axis shows the occurrence frequency.

error bars in the figure. The direction of the perpendicular velocity and the perpendicular temperature do not change significantly around the event. Note that these are average results for all events. The low average values of the two latter parameters also indicate that these values are noisy with significant spikes. As the normalization is done between zero and the maximum value observed in the time window around the event, a few spikes in the data will lower the average value. One may also note that the average increase of the number flux can be deduced from Fig. 4. The average flux increases from a normalized value of 0.25 to 0.55 , i.e. about a $120 \%$ increase.

\subsection{Temperature enhancements}

A histogram of the time between perpendicular temperature enhancement events is shown in Fig. 5. As can be seen there are two clear and distinct peaks. The first is in the two lowest bins. To some extent this is a noise peak, but the perpendicular temperature appears to be very variable. The second peak is at $4 \mathrm{~min}$. The interval between 4 and $7 \mathrm{~min}$ (the local peak in the distribution) contains $28 \%$ of the time intervals between events. The first peak, the 0 to 2 min range, contains another $28 \%$ of the time intervals between temperature enhancement events. The resulting histogram does not depend strongly on the length of the window used (we tried from $30 \mathrm{~s}$ to $300 \mathrm{~s}$ and used $120 \mathrm{~s}$ in the final plot). Neither did the result strongly depend on the threshold value discussed in Sect. 3.1. The result of the superposed epoch analysis of the enhanced temperature events is shown in Fig. 6. That the temperature (red line) shows a clear increase around the transient event time indicates that the detection algorithm works.

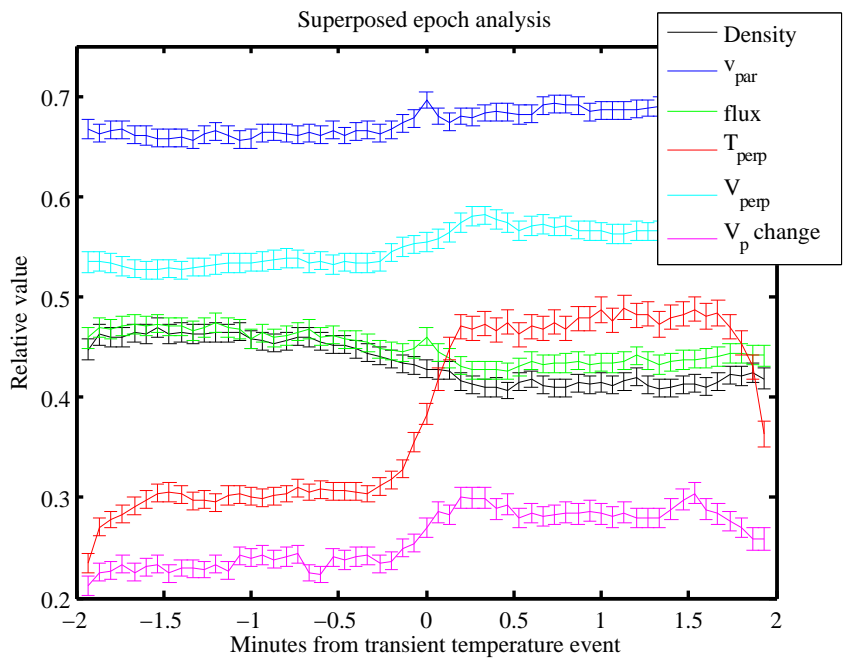

Fig. 6. Superposed epoch analysis of enhanced perpendicular temperature events. For each event the values have been normalized to the range 0 to 1 by dividing with the maximum value in the superposed epoch window of $\pm 2 \mathrm{~min}$. The $\mathrm{x}$-axis shows the time relative to the enhanced temperature events [minutes]. The y-axis shows the normalized value of the following parameters:Black: Density Blue: parallel velocity, Green: number flux, Red: perpendicular temperature, Cyan: magnitude of perpendicular velocity and Magenta: magnitude of vector change of perpendicular velocity.

Of the other parameters the magnitude of the vector change of the perpendicular velocity and the magnitude of the perpendicular velocity show an increase similar to that of the temperature, but just barely above the indicated $95 \%$ confidence limit. The average increase of the perpendicular temperature was from an averaged value of about 0.3 to 0.5 , i.e. an increase of about $70 \%$.

\section{Discussion}

The distribution of the time interval between number flux enhancements showed a clear peak for the time interval 5 to $10 \mathrm{~min}$. This is very similar to what has been reported for cusp transient events and also upflow associated with cusp transient events (Moen et al., 2004). The superposed epoch analysis indicated that the number flux changes were mainly caused by increased densities at the satellite location. Temperature increases were not seen. In the cusp ionosphere one might expect transient changes of the convection to be associated with enhanced upflow. At the satellite altitude we do not see any statistical change of the magnitude or direction of convection around the transient number flux events. One possible explanation for this is the difference in travel time for Alfvén waves (electric field changes) and the outflowing ions. The travel time of the Alfvén waves from the ionosphere to our observation point is typically two to three minutes. The travel time for a $1 \mathrm{keV}$ oxygen ion is about $10 \mathrm{~min}$. We can therefore not expect an experimental confirmation 
from high altitude measurements for the change of the electric field associated with transient events. Higher energy oxygen ions may have velocities closer to the Alfvén wave travel time, but it is not likely that the ions had this high velocity for their entire travel time.

The parallel velocity is on average enhanced in the number flux enhancement events. The contrary is not true, the distribution of parallel velocity enhancements is not at all similar to the distribution of number flux enhancement (not shown). There are many magnetospheric processes accelerating the outflowing ions, but these do not change the amount of observed outflowing ions.

Does the time interval between sudden enhancements as observed at high altitude reflect the time interval between transients at the source? The spacecraft motion is slow compared to both observed convection and observed field aligned velocities, so this does not significantly affect the result. Whether sudden changes reach the spacecraft as a front moving along the field line or as a structure drifting with convection the average time between events should reflect the time between transients at the source. If outflow bursts occur once on each field-line as it moves through a source region (using the frozen in concept of a moving field line) we should see the changes as drifting with convection, there should be no abrupt changes along the field-line. In case studies it has been shown that outflow structures observed in the high altitude polar cap are drifting past the spacecraft with the convection velocity (Nilsson et al., 2004; Maggiolo et al., 2006). For the particular case shown in Fig. 1 this is also the case (not shown). We attempted a statistical cross correlation study to see if this was the case for our sudden number flux enhancements and could not confirm that this is the general case. Often the spacecraft are too close or too far apart to allow for a meaningful cross correlation analysis. If several outflow bursts occur on the same field-line we may see fronts approaching along the field line rather than drifting with convection. Due to velocity dispersion the leading edge of a new outflow burst will overtake the trailing edge of an old outflow burst which may lead to a sudden flux enhancement. The velocity dispersion is also likely to make number flux changes smooth as the particles are spread out along the field-line according to their parallel velocity. Therefore it is likely that the sudden number flux enhancements represent structures drifting past the spacecraft with convection velocity. Our interpretation that the time intervals between sudden enhancements of the number flux corresponds to the time intervals between modulations of the source is not dependent on this assumption. Field-aligned flow will create a larger uncertainty because the parallel velocity of the particles being part of the sudden flux enhancement will affect the time interval between events for a particular case. The average time between events will not be affected. Finally, we see from the superposed epoch analysis that the main reason for the enhancement of the flux is a change of density, not a change of field-aligned velocity.
The distribution of the time interval between perpendicular temperature enhancements was different from that of the number flux enhancements. The temperature enhancements occurred more often, and had peaks in the 0 to $2 \mathrm{~min}$ and in the 4 to $7 \mathrm{~min}$ interval. The distribution peaked at $4 \mathrm{~min}$ interval between the events, and longer time intervals between events were progressively less likely. The superposed epoch analysis did reveal a slight possible interrelation with perpendicular velocity changes. Perpendicular temperature enhancements did not on average cause more particles to be observed by the CODIF instrument, i.e. the observed number flux did not increase.

It is in principle possible that we often have a cold component of the plasma which cannot be observed by CODIF. If these ions are heated or accelerated they may come into the observable energy range of CODIF. The lack of relation between temperature enhancements and number flux indicates that on average this is not an important process. This may be due to the lack of a significant cold population, or because heating is less efficient for cold ions so that the cold core remains cold. For oxygen ions the convection velocity at high altitude will often bring the ions into the observable energy range, which may explain why further energization does not affect the observable number flux at high altitude. The oxygen ion beams typically form a well defined population, well above the observational threshold (see Fig. 1 for an example).

Most indications of the transient nature of the cusp come from ground based observations. Incoherent scatter radars and scanning photometers have shown that transient intensifications in soft electron precipitation and cusp aurora occur with a typical time interval of 5 to $10 \mathrm{~min}$ (Lockwood et al., 1993; Øieroset et al., 1996). Moen et al. (2004) could show a one to one relation between transient cusp aurora (so called Poleward Moving Auroral Forms) and ionospheric upflows. The fact that the high altitude number flux of oxygen ions is modulated in a way similar to what is observed in the ionosphere indicates that the ionospheric upflow is the main limiting factor of the total outflow. It is also worth to point out that whereas many indications on how dynamic the cusp is have come from ground based observations (e.g. Lockwood et al., 1993; Moen et al., 2004), when we study a parameter which is strongly coupled to the ionosphere, the oxygen ion number flux, we do see signs of the same dynamic phenomena in the high altitude spacecraft data.

As to the nature of the transient events we cannot say very much from the high altitude data. As stated above the relation to electric field changes is lost due to differences in travel time between heavy ions and Alfvén waves. Whether it is bursty reconnection, field line resonances or Alfvén wave travel time which causes the periodicity we cannot tell from data obtained far from the source of these transients.

Finally, it is worthwhile to make an order of magnitude comparison of the outflow as observed at high altitude and the ionospheric upflow. The high altitude outflow, normalized to ionospheric altitudes, is about $10^{12}-10^{13} \mathrm{~m}^{-2} \mathrm{~s}^{-1}$, 
using the values from Nilsson et al. (2006), their Fig. 9. Ogawa et al. (2003) reported an upflow flux of typically $10^{11} \mathrm{~m}^{-3} \times 100 \mathrm{~ms}^{-1}=10^{13} \mathrm{~m}^{-2} \mathrm{~s}^{-1}$. This is only a rough comparison, but it does show that the upflow fluxes are similar to the high altitude outflow fluxes. It is therefore worthwhile to compare statistics of the upflow number flux in the cusp region with the outflowing number flux from magnetospheric spacecraft, preferably over a solar cycle.

\section{Conclusions}

Sudden enhancements of the oxygen ion number flux observed at high altitude above the polar cap occur with a typical time interval of 5 to $10 \mathrm{~min}$. This is very similar to the time period between cusp transients believed to be caused by bursts of reconnection (Lockwood et al., 1993). A superposed epoch analysis revealed that the sudden number flux enhancements were not associated with any temperature enhancements in the high altitude magnetosphere. Neither could any clear convection changes be seen in the vicinity of the number flux enhancement. The latter would have been likely, as bursts of reconnection in the ionosphere typically are associated with enhanced convection. We suggest that due to the different propagation speed of Alfvén waves and outflowing oxygen ions any such ionospheric relation will be lost at high altitude.

Sudden enhancements of the oxygen ion perpendicular temperature at high altitude showed a different distribution from that of number flux enhancements. The typical time period between the enhancements was $4 \mathrm{~min}$. The superposed epoch analysis based on the sudden temperature enhancements did not reveal any relation to number flux enhancements.

The above two facts indicate that the ionospheric upflow is the main limiting factor of the oxygen ion outflow. The strongest modulation of the outflow is that which resembles what we expect for ionospheric upflow. There is considerable further energization with a different distribution in the magnetosphere, but this does not affect the observable number flux.

The question on the time scale between number flux enhancements is related to the debate about the dynamics of the cusp (Lockwood et al., 1993; Nilsson et al., 1996). We cannot say from this data how important bursty reconnection is compared to steady reconnection. We cannot even say if the sudden enhancements are caused by bursty reconnection. The time scale is similar to field line eigenmode oscillations at these latitudes and Alfvén wave travel times to and from the ionosphere, so other phenomena may be involved. It seems however that whatever process causes recurring enhancements of the upflow in the ionosphere is an important process for the total oxygen outflow.
Acknowledgements. This work was supported by the National Space Board of Sweden and by the funding agencies of the coauthors.

Topical Editor K. Kauristie thanks two anonymous referees for their help in evaluating this paper.

\section{References}

Andersson, L., Peterson, W. K., and McBryde, K. M.: Estimates of the suprathermal $\mathrm{O}^{+}$outflow characteristic energy and relative location in the auroral oval, Geophys. Res. Lett., 32, L09104, doi:10.1029/2004GL021434, 2005.

Arvelius, S., Yamauchi, M., Nilsson, H., Lundin, R., Hobara, Y., Rème, H., Bavassano-Cattaneo, M. B., Paschmann, G., Korth, A., Kistler, L., and Parks, G. K.: Statistics of high-altitude and high-latitude $\mathrm{O}^{+}$ion outflows observed by Cluster/CIS, Ann. Geophys., 23, 1909-1916, 2005, http://www.ann-geophys.net/23/1909/2005/.

Balogh, A., Carr, C. M., Acuna, M. H., Dunlop, M. W., Beek, T. J., Brown, P., Fornaon, K.-H., Georgescu, E., Glassmeier, K.-H., Harris, J., Musmann, G., Oddy, T., and Schwingenschuh, K.: The Cluster Magnetic Field Investigation: overview of in-flight performance and initial results, Ann. Geophys., 19, 1207-1217, 2001, http://www.ann-geophys.net/19/1207/2001/.

Bouhram, M., Klecker, B., Miyake, W., Rème, H., Sauvaud, J.-A., Malingre, M., Kistler, L., and Blăgău, A.: On the altitude dependence of transversely heated $\mathrm{O}^{+}$distributions in the cusp/cleft, Ann. Geophys., 22, 1787-1798, 2004, http://www.ann-geophys.net/22/1787/2004/.

Dubouloz, N., Delcourt, D., Malingre, M., Berthrlier, J.-J., and Chugunin, D.: Remote analysis of cleft ion acceleration using thermal plasma measurements from Interball Auroral Probe, Geophys. Res. Lett., 25, 2925-2928, 1998.

Elphic, R. C., Lockwood, M., Cowley, S. W. H., and Sandholt, P. E.: Flux transfer events at the magnetopause and in the ionosphere, Geophys. Res. Lett., 17, 2241-2344, 1990.

Knudsen, D. J., Whalen, B., Abe, T., and Yau, A.: Temporal evolution and spatial dispersion of ion conics: Evidence for a polar cap heating wall, in: Solar System Plasmas in Space and Time, Geophysical Monograph series, Temporal evolution and spatial dispersion of ion conics: Evidence for a polar cap heating wall, edited by: Burch, J. L. and Waite Jr., J. H., vol. 84, p. 163-169, AGU (American Geophysical Union), 1994.

Lockwood, M., Waite Jr., J. H., Moore, T. E., Chappell, C. R., and Johnson, J. F. E.: A new source of Suprathermal O ${ }^{+}$ions near the dayside polar cap boundary, J. Geophys. Res., 90, 4099-4116, 1985.

Lockwood, M., Denig, W. F., Farmer, A. D., Davda, V. N., Cowley, S. W. H., and Lühr, H.: Ionospheric signatures of pulsed reconnection at the Earth's magnetopause, Nature, 361, 424-428, 1993.

Maggiolo, R., Sauvaud, J. A., Fontaine, D., Teste, A., Grigorenko, E., Balogh, A., Fazakarley, A., Paschmann, G., and Rème, H.: A multi-satellite study of accelerated ionospheric ion beams above the polar cap, Ann Geophys., 24, 1665-1684, 2006.

Moen, J., Oksavik, K., and Carlson, H. C.: On the relationship between ion upflow events and cusp auroral transients, Geophys. Res. Lett., 31, L11808, doi:10.1029/2004GL020129, 2004. 
Nilsson, H., Kirkwood, S., Eliasson, L., Norberg, O., Clemmons, J., and Boehm, M.: The ionospheric signature of the cusp: A case study using Freja and the Sondrestrom radar, Geophys. Res. Lett., 21, 1923-1926, 1994.

Nilsson, H., Yamauchi, M., Eliasson, L., Norberg, O., and Clemmons, J.: The ionospheric signature of the cusp as seen by incoherent scatter radar, J. Geophys. Res., 101, 10 947-10 963, 1996.

Nilsson, H., Kirkwood, S., and Moretto, T.: Incoherent scatter radar observations of the cusp acceleration region and cusp fieldaligned currents, J. Geophys. Res., 103, 26721-26730, 1998.

Nilsson, H., Joko, S., Lundin, R., Rème, H., Sauvaud, J.-A., Dandouras, I., Balogh, A., Carr, C., Kistler, L. M., Klecker, B., Carlson, C. W., Bavassano-Cattaneo, M. B., and Korth, A.: The structure of high altitude $\mathrm{O}^{+}$energization and outflow: a case study, Ann. Geophys., 22, 2497-2506, 2004, http://www.ann-geophys.net/22/2497/2004/.

Nilsson, H., Waara, M., Arvelius, S., Marghitu, O., Bouhram, M., Hobara, Y., Yamauchi, M., Lundin, R., Rème, H., Sauvaud, J.A., Dandouras, I., Balogh, A., Kistler, L. M., Klecker, B., Carlson, C. W., Bavassano-Cattaneo, M. B., and Korth, A.: Characteristics of high altitude oxygen ion energization and outflow as observed by Cluster; a statistical study, Ann. Geophys., 24, 1099-1112, 2006,

http://www.ann-geophys.net/24/1099/2006/.

Norqvist, P., André, M., and Tryland, M.: A statistical study of ion energization mechanisms in the auroral region, J. Geophys. Res., 103, 23 459-23 474, 1998.

Ogawa, Y., Fujii, R., Buchert, S. C., Nozawa, S., and Ohtani, S.: Simultaneous EISCAT Svalbard radar and DMSP observations of ion upflow in the dayside polar ionosphere, J. Geophys. Res., 108, 1101, doi:10.1029/2002JA009590, 2003.

Øieroset, M., Lühr, H., Moen, J., Moretto, T., and Sandholt, P. E.: Dynamic auroral morphology in relation to ionospheric plasma convection and geomagnetic activity: Signatures of magnetopause X line dynamics and flux transfer events, J. Geophys Res., 101, 13 275-13 292, 1996.
Øieroset, M., Yamauchi, M., Liszka, L., Christon, S. P., and Hultqvist, B.: A statistical study of ion beams and conics from the dayside ionosphere during different phases of a substorm, J. Geophys. Res., 104, 6987-6998, 1999.

Rème, H., Aoustin, C., Bosqued, J. M., Dandouras, I., Lavraud, B., Sauvaud, J. A., Barthe, A., Bouyssou, J., Camus, T., Coeur-Joly, O., Cros, A., Cuvilo, J., Ducay, F., Garbarowitz, Y., Medale, J. L., Penou, E., Perrier, H., Romefort, D., Rouzaud, J., Vallat, C., AlcaydE, D., Jacquey, C., Mazelle, C., d'Uston, C., Möbius, E., Kistler, L. M., Crocker, K., Granoff, M., Mouikis, C., Popecki, M., Vosbury, M., Klecker, B., Hovestadt, D., Kucharek, H., Kuenneth, E., Paschmann, G., Scholer, M., Sckopke, N., Seidenschwang, E., Carlson, C. W., Curtis, D. W., Ingraham, C., Lin, R. P., McFadden, J. P., Parks, G. K., Phan, T., Formisano, V., Amata, E., Bavassano-Cattaneo, M. B., Baldetti, P., Bruno, R., Chionchio, G., Lellis, A. D., Marcucci, M. F., Pallocchia, G., Korth, A., Daly, P. W., Graeve, B., Rosenbauer, H., Vasyliunas, V., McCarthy, M., Wilber, M., Eliasson, L., Lundin, R., Olsen, S., Shelley, E. G., Fuselier, S., Ghielmetti, A. G., Lennartsson, W., Escoubet, C. P., Balsiger, H., Friedel, R., Cao, J.-B., Kovrazhkin, R. A., Papamastorakis, I., Pellat, R., Scudder, J., and Sonnerup, B.: First multispacecraft ion measurements in and near the Earth's magnetosphere with the identical Cluster ion spectrometry (CIS) experiment, Ann. Geophys., 19, 1303-1354, 2001, http://www.ann-geophys.net/19/1303/2001/.

Schunk, R. W., Raitt, W. J., and Banks, P. M.: Effect of electric fields on the daytime high-latitude $E$ and $F$ regions, J. Geophys. Res., 80, 3121-3130, 1975.

Yau, A. W. and André, M.: Sources of ion outflow in the high latitude ionosphere, Space Sci. Rev., 80, 1-25, 1997. 